\title{
Wavelet Transform based CT and PET Lung Image Fusion
}

\author{
M. Chithra Devi
}

\begin{abstract}
The lung cancer reduces the breathing ability and it causes due to uncontrollable cells in the lungs. The fusion of Computed Tomography (CT) and Positron Emission Tomography (PET) has clear information about lungs comparing with individual CT and PET images. The fused image helps to find cancer in a starting stage. In this study, a novel method for fusion of CT and PET lung images using Wavelet Transform (WT) and MaxMin (MM) rule is presented. Initially, the CT and PET lung images are decomposed by WT. The subband coefficients of WT are extracted by using entropy features. Then MM rule is applied, after that the image is reconstructed to form the fused lung image. The performance is evaluated by Reference Image Database to Evaluate therapy Response (RIDER) lung database. Results and discussion shows the performance of Lung Image Fusion (LIF).
\end{abstract}

Keywords : Lung Image Fusion, Wavelet Transform, Maxmim Rule, RIDER Database.

\section{INTRODUCTION}

LIF based on structural similarity index and max rule is discussed in [1]. Initially, the CT and PET images are divided into different blocks. The CT block corresponds to the PET block. Then structural similarity index is applied to form the fused lung image. The thresholding technique is used to detect the lung nodule. Wavelet denoising and image fusion based ribs segmentation is discussed in [2]. The lung parenchyma was extracted by using Otsu method. Then the lung fusion is made by wavelet image fusion algorithm. The multiscale wavelet is applied for noise removal. The Gaussian mask is used for the segmentation and smoothened the ribs.

Lung fusion based on lung vessel registration is discussed in [3] using CT and magnetic resonance images. The perfusion analysis and lung vessel segmentation is performed for both images. The pixel intensity is adjusted, the feature point is also determined, and then finally the elastic matching is made to form a fused image. Lung fusion based on three-dimensional Convolutional Neural Network $(\mathrm{CNN})$ is described in [4]. CNN is used for the LIF. The hu unit is used for the segmentation of lung images.

CT and PET LIF based on supervised co-segmentation is described in [5]. The input lung image features are extracted by using computational complexity. The cost for mono-modality, global cost function, cost for inconsistency and iterative optimization is used for lung image segmentation. Dual tree complex wavelet transform based PET-CT LIF is discussed in [6]. At first, the pretreatment and registration is used for the preprocessing technique, the higher and lower frequency components of dual-tree complex

Revised Manuscript Received on December 12, 2019.

* Correspondence Author

First Author Name*, Dr.M.Chithra Devi, Assistant Professor and Head in Department of Information Technology, Annai Vailankanni Arts and Science College (Affiliated to Bharathidasan University), Thanjavur, India. Email: m.chithradevi@gmail.com wavelet transform is used to form the fused image. The image reconstruction is also made. Finally, the fused image is obtained. Multistage fusion approach based on non-small cell lung cancer is discussed in [7]. The input CT and PET images are fused by using fuzzy clustering means, the ensemble clustering methods and fusion.

$\mathrm{CNN}$ based feature fusion and lung disease classification is described in [8]. The lung images are preprocessed at first. The LIF is made by using CNN and Gabor filters with enhanced information. Lung tumor classification based on multi layer perceptron is discussed in [9]. Initially, the lung nodules are segmented by gray level co-occurrence matrix. The features like homogeneity, mean, standard deviation, angular second moment, energy and standard deviation are extracted. The classification is made by multilayer perceptron and k-nearest neighbor. Lung cancer detection in CT lung images is discussed in [10]. Initially, the lung images are preprocessed by using image preprocessing and median filter The region of interest is extracted by using morphological operations. Then the features are extracted by geometrical features. Support vector machine classifier is used for classification.

A novel method for CT and PET LIF using wavelet transform and maxmin rule is discussed in this study. The organization of the paper is as follows: Section 2 describes the methods and materials used for CT and PET LIF. In section 3 the results and discussion of lung fusion is described. The last section concludes the CT and PET LIF.

\section{METHODS AND MATERIALS}

LIF using CT and PET lung images based on WT and MM is shown in figure 1. Initially the lung images are decomposed by WT and it produces the subband coefficients. Then the entropy features are extracted. Finally, MM rule is applied to perform the LIF.

\section{A. WT Decomposition}

The different merit function in WT and fourier transform is similar to each other. WT uses the fourier and real space. WT is also used in object recognition [11], brain image classification [12] and weld image classification [13]. It returns the data vector with the same input length. WT decomposes into a set of wavelets which has the orthogonal of scaling and translations. The decomposition of WT has the compression of the wavelet spectrum in some data points. It is defined by,

$$
K(u, v)=\int_{-\infty}^{\infty} k(g) \xi_{(u, v)}^{*}(g) d g
$$


where $\xi$ is some function and $*$ is the conjugate symbol. The certain rules are performed under this function. In this study, the WT is used to decompose the CT and PET lung images and it produces the subband coefficients.

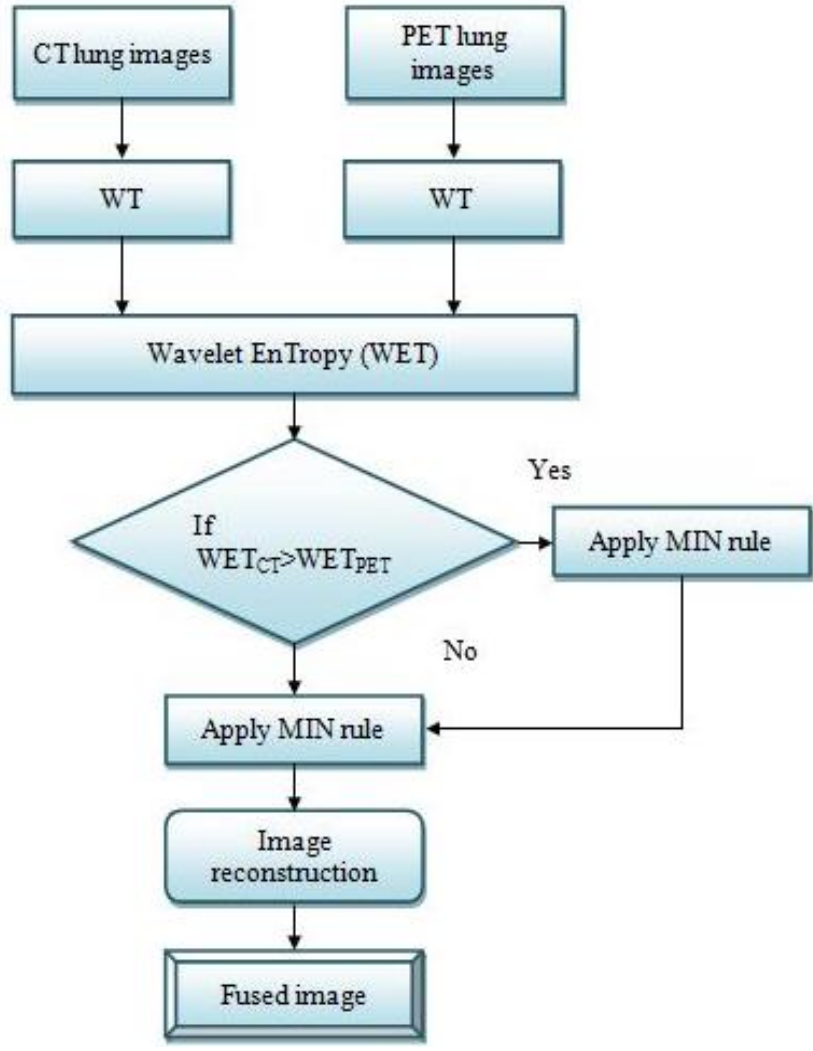

Fig. 1.CT and PET LIF using WT and MM

\section{B. WT based entropy feature extraction}

The information content and statistical measurement of the input in the uncertainty degree is known as entropy. The information measurement is known as entropy, it adopts the image to consider the image as a specific image. Entropy feature is also used in palm print recognition [14] and glaucoma image classification [15]. The subband is coded in each image and also it measures the quantity of the image. The WET is defined by,

$$
W E T=-\sum_{j} G_{j} \log _{2}\left(G_{j}\right)
$$

where $G_{j}$ is the probability $j$ is the level of probability and the subband information is denoted as $-\log _{2}$. In this study, the WT coefficients are extracted by using entropy features.

\section{MM Rule}

It is the essential tool for decomposition in fuzzy logic rules and wavelet analysis. MM rule is frequently used in the lower and higher frequency components. The subproblem finds the min unit for the two class subproblem which is presented in the basic units. The min unit finds the minimum value for given inputs and max units finds the maximum value for the inputs. The two class subproblem for MM rule is defined by,

$$
\begin{gathered}
S_{a, b}^{u}=\operatorname{MIN}\left(S_{a, b}^{u, 1}, S_{a, b}^{u, 2}, \ldots, S_{a, b}^{u, K b}\right) \\
S_{a, b}=\operatorname{MAX}\left(S_{a, b}^{1}, S_{a, b}^{2}, \ldots . S_{a, b}^{K a}\right)
\end{gathered}
$$

Where

$$
u=1,2,3, \ldots . S_{a}
$$

$S_{a, b}^{u, 1}\left(u=1,2, \ldots \ldots, \quad K_{a}, b=1,2, \ldots ., S_{a}\right)$ is the base classifier output. In this study, MM rule is used for the CT and PET LIF. If PET value is less that CT apply min rule otherwise apply max rule. Then reconstruct the image to form the fused image.

\section{RESULTS AND DISCUSSION}

The LIF performance is evaluated by using RIDER database [17-18]. Some sample lung CT and PET images in RIDER database are shown in figure 2.

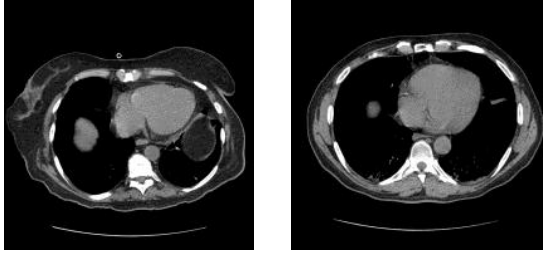

(a) Lung images-CT

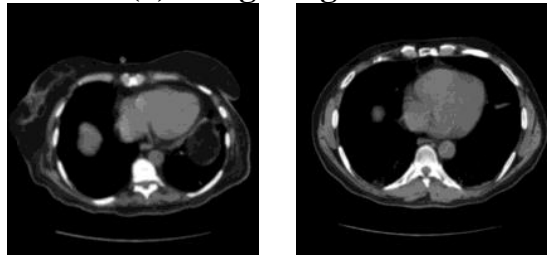

(b) Lung images-PET

Fig. 2.Sample lung images in the RIDER database

At first, the input CT and PET lung images are given to WT and it produces lower and higher frequency subband coefficients. These subband coefficients are extracted by entropy features. Then the MM rule is applied for the fusion of CT and PET lung images. If WETCT is greater than WETPET apply min rule otherwise apply max rule. Then the inverse transform is applied to reconstruct the image. Finally fused mage is obtained by using WT and MM rule. Figure 3 shows the fused image obtained by using WT and MM rule.

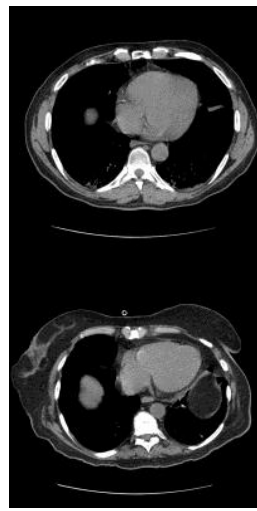

(a) CT lung image

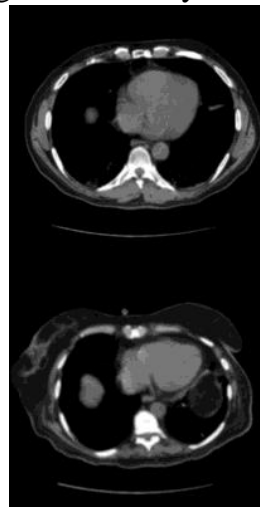

(b) PET lung image

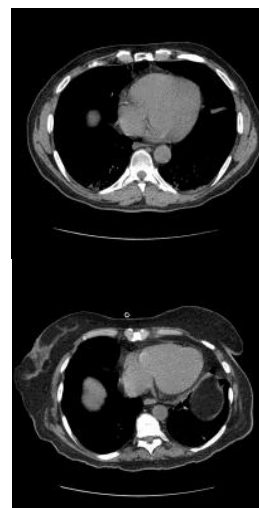

(c) Fused lung image
Fig. 3.Performance of fusion using WT and MM rule

The performance of LIF is measured by Peak Signal to Noise Ratio (PSNR) values. The PSNR is measured by mean square error. The PSNR is measured by,

$$
P S N R=10 \log _{10}\left(\frac{P^{2}}{L}\right)
$$

where $P$ is the maximum function of input, and $L$ is a mean square error. Table 1 shows the performance of PSNR values for LIF using some sample images in the RIDER database.

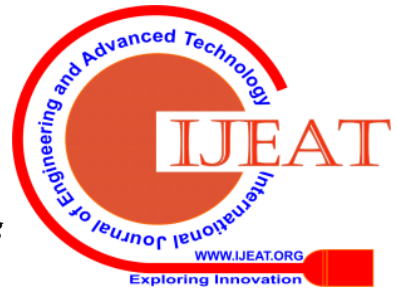


Table- II: Performance of PSNR values for LIF

\begin{tabular}{|c|c|c|}
\hline \multirow{2}{*}{$\begin{array}{c}\text { Sample images } \\
\text { S.No. }\end{array}$} & \multicolumn{2}{|c|}{ PSNR Performance } \\
\cline { 2 - 3 } & CT (dB) & PET (dB) \\
\hline 1 & 61.43 & 31.24 \\
\hline 2 & 64.79 & 41.53 \\
\hline 3 & 62.70 & 38.79 \\
\hline 4 & 58.61 & 35.54 \\
\hline 5 & 64.38 & 40.80 \\
\hline
\end{tabular}

From Table 1 it is observed that the performance of PSNR is approximately $64.79 \mathrm{~dB}$ for CT images and $41.53 \mathrm{~dB}$ for PET images. It gives the best fusion performance by using WT and MM rule.

\section{CONCLUSION}

A novel method for LIF using WT and MM rule is presented in this paper. The input lung images are decomposed by WT to produce lower and higher frequency subband coefficients. These subband coefficients are extracted by entropy features and given to MM rule. If CT image value is greater than PET image value the min rule is applied otherwise max rule is applied. The inverse transform is applied to reconstruct the image and forms the LIF. Finally, the CT, PET and fused lung image is measured by using PSNR values for better performance using WT and MM rule.

\section{REFERENCES}

1. P. Venkatesan, "Image Fusion Based Lung Nodule Detection Using Structural Similarity And Max Rule", International Journal of Advances in Signal and Image Sciences, Vol. 5, No. 1, 2019, pp. 29-35.

2. W. Cui, T. Yu, L. Ren, and H. Shao, "Ribs segmentation based on image fusion and wavelet de-noising", International Conference on BioMedical Engineering and Informatics, 2012, pp. 362-366.

3. Y. Iwao, Y. Wei, S. Kagei, T. Gotoh, T. Iwasawa, and M.D.S.G. Tsuzuki, "Fusion of lung MR/CT images through lung vessel registration", International Conference of the IEEE Engineering in Medicine and Biology Society, 2013, pp. 3419-3422.

4. G. Cao, T. Huang, K. Hou, W. Cao, P. Liu, and J. Zhang, "3D Convolutional Neural Networks Fusion Model for Lung Nodule Detection onClinical CT Scans", IEEE International Conference on Bioinformatics and Biomedicine, 2018, pp. 973-978.

5. C. Lian, H. Li, P. Vera, and S. Ruan, "Unsupervised co-segmentation of tumor in PET-CT images using belief functions based fusion", International Symposium on Biomedical Imaging, 2018, pp. 220-223

6. X. Wei, T. Zhou, and H. Lu, "A fusion algorithm of PET-CT based on dual-tree complex wavelet transform and self-adaption Gaussian membership function", International Conference on Orange Technologies, 2014, pp. 216-219.

7. M.W. Farouq, W. Boulila, M. Abdel-Aal, A. Hussain, and A.B. Salem, "A Novel Multi-Stage Fusion based Approach for Gene Expression Profiling in Non-Small Cell Lung Cancer”, IEEE Access, Vol. 7, 2019, pp. 37141-37150.

8. Y. Cheng, J. Feng, and K. Jia, “A Lung Disease Classification Based on Feature Fusion Convolutional Neural Network with X-ray Image Enhancement", Asia-Pacific Signal and Information Processing Association Annual Summit and Conference, 2018, pp. 2032-2035.

9. S. Potghan, R. Rajamenakshi, and A. Bhise, "Multi-Layer Perceptron Based Lung Tumor Classification", International Conference on Electronics, Communication and Aerospace Technology, 2018, pp. 499-502.

10. N.S. Nadkarni, and S. Borkar, "Detection of Lung Cancer in CT Images using Image Processing", International Conference on Trends in Electronics and Informatics, 2019, pp. 863-866.

11. A. Jeyasudha, and K. Priya, "Object recognition based on LBP and discrete wavelet transform", International journal of advances in signal and image sciences, Vol. 2, No. 1, 2016, pp. 24-30.

12. S. Mohankumar, "Analysis of different wavelets for brain image classification using support vector machine", International Journal of Advances in Signal and Image Sciences, Vol. 2, No. 1, 2016, pp. 1-4.
13. V. Kalaiselvi, and D.J. Aravindhar, "An Efficient Weld Image Classification System Using Wavelet And Support Vector Machine", International Conference on Computing and Communications Technologies, 2019, pp. 46-49.

14. S. Afsal, J. Jothykumar, S. Ahmed, and F. Sayeed, "A novel approach for palm print recognition using entropy information features", International Conference on Wireless Communications, Signal Processing and Networking, 2016, pp. 1439-1442.

15. B.S. Kirar, and D.K. Agrawal, "Empirical wavelet transform based pre-processing and entropy feature extraction from glaucomatous digital fundus images" International Conference on Recent Innovations in Signal processing and Embedded Systems, 2017, pp. 315-319.

16. Y. Yang, and W.K. Tsai, "A Maxmin Routing Algorithm for Dense Wavelength Division Multiplexing Networks", International Conference on Communication Technology, 2006, pp. 1-4.

17. K. Clark, B. Vendt, K. Smith, J. Freymann, J. Kirby, P. Koppel, S. Moore, S. Phillips, D. Maffitt, M. Pringle, and L. Tarbox, "The Cancer Imaging Archive (TCIA): maintaining and operating a public information repository", Journal of digital imaging, Vol. 26, No. 6, 2013, pp.1045-1057.

18. http://doi.org/10.7937/K9/TCIA.2015.OFIP7TVM

\section{AUTHORS PROFILE}

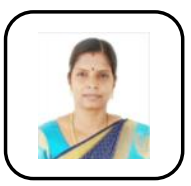

Dr.M.Chithra Devi, Assistant Professor and Head in Department of Information Technology, Annai Vailankanni Arts and Science College, Thanjavur. She received best paper award for "Pair of Iris Recognition using Feedforward Neural Networks" 2nd International Conference on Advances in Engineering and Technology -ICAET in collaboration with Aichi Institute of Technology -Toyota, 2012 at E.G.S. Pillay Engineering College, Nagapattinam in 2012.She has completed her Under Graduate Degree in Information Technology at Ponnaiyah Ramajayam College in 2004. Post Graduate Degree in Computer Science at T.U.K. Arts College, Thanjavur in 2006 and Master of Philosophy in Computer Science at A.V.V.M.Sri Pushpam College, Poondi in 2007. She completed her Master of Engineering in computer science at Periyar Maniammai University, Thanjavur in 2012. Ph.D in Computer Science at Bharathiar University, Coimbatore and awarded in the year of 2019. She has twelve years experience. She published more than 12 research articles of various domains in peer reviewed International Journals 